\title{
Correlação entre sintomas osteomusculares e qualidade de vida de professores do ensino fundamental
}

\author{
Correlation between musculoskeletal symptoms and quality of life of \\ elementary and middle school teachers
}

\section{Correlación entre síntomas osteomusculares y calidad de vida de los profesores de educación fundamental}

\author{
Fernandes, Geyse Chrystine Pereira Souza ${ }^{1}$ (Guanambi, BA, Brasil) \\ ORCID ID: https://orcid.org/0000.0003.2339.8878 \\ Almeida, Rogério José de ${ }^{2}$ (Goiânia, GO, Brasil) \\ ORCID ID: https://orcid.org/0000.0002.2150.6057
}

\begin{abstract}
Resumo
O estudo objetivou analisar a correlação entre os sintomas osteomusculares e a qualidade de vida de professores do ensino fundamental da rede pública municipal de Guanambi, Bahia. Trata-se de estudo transversal analítico em que 52 professores do ensino fundamental participaram. Foram utilizados os instrumentos: Questionário sociodemográfico ocupacional, Questionário Nórdico de Sintomas Osteomusculares (QNSO), Instrumento de avaliação da qualidade de vida - WHOQOL-Bref. O perfil sociodemográfico ocupacional demonstrou predominância do sexo feminino, a maioria com jornada de trabalho de 40 horas semanais e possuindo pós-graduação. Uma grande maioria entendeu que seu trabalho tem risco à saúde e que ainda se cansa com frequência. Houve alta prevalência de sintomas osteomusculares, principalmente em joelho, coluna lombar, ombro e pescoço. Entre os sintomas osteomusculares em correlação ao perfil sociodemográfico ocupacional houve relação significativa entre $o$ afastamento e se sentir cansado no trabalho. No Whoqol-bref a qualidade de vida total, a percepção da qualidade de vida e a satisfação pessoal apresentaram boas médias. A qualidade de vida total e o domínio físico foram afetados em todos os resultados de correlação com os sintomas osteomusculares. Constatou-se que os sintomas osteomusculares afetam diretamente a qualidade de vida dos professores.
\end{abstract}

Palavras-Chave: professor; qualidade de vida; transtornos traumáticos cumulativos.

\begin{abstract}
The aim of this study was to analyze the correlation between musculoskeletal symptoms and the quality of life of primary school teachers in the municipal public network of Guanambi, Bahia. This is an analytical cross-sectional study in which 52 elementary school teachers participated. The following instruments were used: Occupational sociodemographic questionnaire, Nordic Questionnaire of Musculoskeletal Symptoms (QNSO), Quality of Life Assessment Tool - WHOQOL-Bref. The occupational sociodemographic profile showed a predominance of females, most of them with a 40 hour workweek and a post-graduate degree. A large majority understood that their work is at risk to health and that they still tire frequently. There was a high prevalence of musculoskeletal symptoms, mainly in the knee, lumbar spine, shoulder, and neck. Among the musculoskeletal symptoms in correlation with the occupational sociodemographic profile there was a significant relation between withdrawal and feeling tired at work. In the Whoqol-bref the total quality of life, the perception of the quality of life and the personal satisfaction presented good averages. Total quality of life and physical domain were affected in all results correlating with musculoskeletal symptoms. It was found that musculoskeletal symptoms directly affect teachers' quality of life.
\end{abstract}

Keywords teacher; quality of life; cumulative traumatic disorders.

\footnotetext{
1 Mestre em Ciências Ambientais e Saúde pela Pontifícia Universidade Católica de Goiás (PUC Goiás). geysefisioterapia@gmail.com

2 Professor Adjunto do Curso de Medicina da PUC Goiás e do Programa de Pós-Graduação em Ciências Ambientais e Saúde - PPGCAS da PUC Goiás. rogeriopucgo@gmail.com
} 


\section{Resumen}

El estudio tuvo como objetivo analizar la correlación entre los síntomas musculoesqueléticos y la calidad de vida de los maestros de primaria en la escuela pública municipal de Guanambi, Bahía. Este es un estudio analítico transversal en el que participaron 52 maestros de primaria. Se utilizaron los siguientes instrumentos: cuestionario sociodemográfico ocupacional, cuestionario musculoesquelético nórdico (QNSO), instrumento de evaluación de la calidad de vida - WHOQOL-Bref. El perfil sociodemográfico ocupacional mostró un predominio de mujeres, la mayoría de ellas trabajando 40 horas a la semana y con títulos de posgrado. Una gran mayoría entendió que su trabajo está en riesgo para la salud y que todavía se cansan con frecuencia. Hubo una alta prevalencia de síntomas musculoesqueléticos, especialmente en la rodilla, columna lumbar, hombro y cuello. Entre los síntomas musculoesqueléticos en correlación con el perfil sociodemográfico ocupacional, hubo una relación significativa entre el permiso y sentirse cansado en el trabajo. En Whoqol-bref, la calidad de vida total, la percepción de la calidad de vida y la satisfacción personal mostraron buenos promedios. La calidad de vida total y el dominio físico se vieron afectados en todos los resultados de la correlación con los síntomas musculoesqueléticos. Se encontró que los síntomas musculoesqueléticos afectan directamente la calidad de vida de los maestros.

Palabras clave: profesor; calidad de vida; trastornos traumáticos acumulativos.

\section{Introdução}

O trabalho faz parte das necessidades humanas para que o homem seja mais livre e mais autônomo. Ao mesmo tempo pode ser considerado como um elemento de subordinação, de sacrifício e mortificação do homem. Assim, o trabalho se constitui na própria finalidade da vida (BUENO, 2015).

O ritmo acelerado da produção capitalista e os efeitos das condições de trabalho determinam, muitas vezes, prejuízos à saúde do trabalhador. Assim, é evidente 0 aparecimento de doenças relacionadas diretamente ao trabalho, principalmente os distúrbios musculoesqueléticos, que afetam a saúde física e mental dos trabalhadores (ANDRADE, 2014).

As áreas médicas e de saúde ocupacional utilizam os distúrbios osteomusculares relacionados ao trabalho (DORT) para designar um conjunto de alterações osteomusculares sofridas pelos trabalhadores em suas atividades laborais (MARINHO, 2012). Tais distúrbios vêm se tornando a principal causa de afastamento de trabalhadores em todos os países, correspondendo em uma epidemia mundial, mesmo que a subnotificação colabore para a falta do conhecimento da situação real (COSTA; FLAUSINO, 2015).

Dessa forma os distúrbios do sistema musculoesquelético têm se tornado foco de pesquisadores preocupados com questões relativas à saúde do trabalhador, devido a seu impacto financeiro e na qualidade de vida, já que esses distúrbios incluem várias doenças articulares, problemas de coluna, condições ósseas e trauma com difícil avaliação clínica (COSTA; FLAUSINO, 2015).

O trabalho do professor é um campo interdisciplinar que associa aspectos 


\section{Revista Labor}

Programa de Pós-graduação em Educação, Universidade Federal do Ceará

Fortaleza-CE-Brasil

organizacionais, produtivos, psicológicos e sociais (CORTEZ et al, 2017). Nos últimos anos, as condições de trabalho dos professores têm se tornado cada vez mais um problema em muitos países. Essas condições enfrentadas na rotina diária do professor podem levar a um desequilíbrio entre o trabalho e a saúde física e mental (CEZAR-VAZ et al., 2015). Dentre as consequências estão as doenças musculoesqueléticas que podem afetar diretamente a qualidade de vida desses profissionais (MOREIRA, 2015).

A definição de qualidade de vida da Organização Mundial da Saúde (OMS) evidencia a percepção subjetiva dos diversos aspectos da vida, como o estado físico psicológico, meio ambiente e relações sociais. Para a OMS, a qualidade de vida se refere "a percepção que um indivíduo tem sobre a sua posição na vida, dentro do contexto dos sistemas de cultura e valores nos quais está inserido e em relação aos seus objetivos, expectativas, padrões e preocupações" (OMS, 1995, p. 1405).

As pesquisas das últimas décadas têm aprimorado o conceito de qualidade de vida, considerando as áreas de saúde, sociais, ambientais e individuais, em relação ao bem-estar da vida coletiva e individual, com abordagens interdisciplinares que perpassam por uma construção subjetiva e multidimensional da vida (VASCONCELOS et al., 2020).

Diante disso é salutar investigar o campo de saúde do trabalho docente para, assim, contribuir na melhoria das condições de trabalho dos professores, na qualidade da educação e no desenvolvimento da saúde coletiva (CORTEZ et al, 2017). Nesse sentido, o presente estudo teve por objetivo analisar a correlação entre os sintomas osteomusculares e a qualidade de vida de professores do ensino fundamental da rede pública municipal de Guanambi, Bahia.

\section{Métodos}

O método apresentado trata-se de um estudo transversal analítico com abordagem quantitativa. Utiliza-se de linguagem matemática e busca descrever as causas de um fenômeno e as relações entre as variáveis, recolhidos com o auxílio de instrumentos padronizados (GERHARDT; SILVEIRA, 2009). É um corte instantâneo de uma população por meio de amostragem em que expõe o fator ou causa presente (HOCKMAN, 2005).

A pesquisa foi realizada com professores do ensino fundamental do 


\section{Revista Labor}

Programa de Pós-graduação em Educação, Universidade Federal do Ceará

Fortaleza-CE-Brasil

municipio de Guanambi/BA. Foram pesquisados os professores que estavam exercendo suas funções docentes como servidor público estável, pertencentes ao quadro de funcionários municipais. A cidade de Guanambi está situada na região sudoeste da Bahia com população estimada para 2017 (IBGE, 2017) em 86808 habitantes, sendo o vigésimo município mais populoso da Bahia.

Inicialmente foi solicitada a autorização da Secretaria de Educação para a realização da pesquisa, a qual obteve resposta positiva. As visitas foram realizadas entre os meses de fevereiro a maio de 2017. A pesquisa foi apresentada aos professores, juntamente com o Termo de Consentimento Livre e Esclarecido, explicando os critérios de inclusão e exclusão.

Os critérios de inclusão foram: professores do ensino fundamental (compreendem do primeiro ao nono ano); professores com no mínimo 10 anos de exercício contínuo na profissão docente; que fosse somente servidor público municipal durante toda a docência.

Os critérios de exclusão foram: professores que trabalhavam em outra instituição, além das escolas municipais; professores que, no período de coleta de dados exerciam outras funções que não a de docência (por exemplo, funções administrativas) e aqueles que se encontravam afastados de suas atividades.

O município possuía 293 professores efetivos no ensino fundamental do $1^{\circ}$ ao 9a ano. Encontravam-se afastados da sala de aula, segundo a Secretaria de Educação, 79 professores, restando 214 indivíduos, onde 55 professores responderam aos questionários, porém 3 foram descartados porque não estavam completos, restando 52 indivíduos, representando uma amostragem por conveniência.

Para o desenvolvimento da pesquisa foi elaborado um questionário sociodemográfico ocupacional para identificar dados como: idade, sexo, estado civil, filhos, religião, formação acadêmica, renda, carga horária, atividade física, atividades domésticas, uso de computador, cansaço no ambiente e satisfação no trabalho.

Foi aplicado o Questionário Nórdico de Sintomas Osteomusculares, um instrumento reconhecido internacionalmente para avaliação de queixas musculoesqueléticas. O objetivo da escala é avaliar problemas musculoesqueléticos em uma abordagem ergonômica, além de padronizar a mensuração de relato de sintomas osteomusculares, facilitando a comparação dos resultados entre os estudos. 


$$
\text { E uma escala que consiste em questóes que consideram as partes do }
$$

corpo humano em nove áreas anatômicas (três para os membros superiores, três para os membros inferiores e três para as costas). As questões são binárias (sim ou não) e se referem à ocorrência dos sintomas considerando os 12 meses e os sete dias anteriores à entrevista, bem como relatar a ocorrência de afastamento das atividades rotineiras no último ano (PINHEIRO et al, 2002).

O instrumento foi adaptado para a língua portuguesa por Barros e Alexandre em 2003, apresentando uma confiabilidade variando de 0,88 a 1 (coeficiente de Kappa). Isso garante que o questionário possa ser aplicado aos trabalhadores brasileiros e que os resultados serão confiáveis, podendo ser utilizados para fornecer dados padronizados para a prática clínica, pesquisa e programas de saúde pública (BARROS; ALEXANDRE, 2003).

Os escores são obtidos para cada um dos fatores da escala sendo eles: os últimos 12 meses, últimos sete dias e afastamento; variando de nove (nenhum sintoma) a 24. Quanto maior o escore, maior é a ocorrência de sintomas osteomusculares nas variadas partes do corpo do indivíduo. Já para os escores de dor a variação é de zero (sintoma sem nenhuma dor) a dez (sintoma com dor máxima).

Também foi aplicado um instrumento de avaliação da qualidade de vida - O WHOQOL-Bref, desenvolvido pela Organização Mundial de Saúde (OMS), adaptado e validado no Brasil por Fleck et al (2000) busca avaliar os indivíduos dentro de uma perspectiva transcultural e internacional (FLECK et al, 2000).

O instrumento é constituído de 26 perguntas (sendo as perguntas número 1 e 2 sobre a autopercepção do entrevistado de sua qualidade de vida). As respostas seguem uma escala de Likert (de 1 a 5, quanto maior a pontuação melhor a qualidade de vida). As demais 24 questões são subdivididas em quatro domínios: físico, psicológico, relações sociais e meio ambiente (FLECK et al, 2000).

O WHOQOL-Bref foi avaliado a partir de uma construção proposta pelo "WHOQOL Group" (WHOQOL GROUP, 1998). Em que foram invertidos os valores da escala para as facetas 3, 4 e 26; em seguida, agruparam-se as facetas em domínios; depois foram calculados os escores de cada domínio (de 1 a 5, por meio da média).

Para análise e entendimento dos escores de qualidade de vida do WHOQOL-Bref foram levadas em consideração as seguintes categorias de resultados: de 1 a 1,99 necessita melhorar; de 2 a 2,99 regular; de 3 a 3,99 boa; e de 
As análises estatísticas foram realizadas com o auxílio pacote estatístico SPSS 24 (Statistical Package of Social Sciences). A caracterização do perfil sociodemográfico foi realizado por meio de frequência absoluta $(n)$ e relativa (\%) $A$ normalidade dos dados foi verificada utilizando o teste de Shapiro-Wilk. Não sendo verificado esse pressuposto foram realizadas estatísticas não paramétricas. A comparação dos escores dos sintomas osteomusculares (Nórdico) e qualidade de vida (WHOQOL-Bref) com o perfil sociodemográfico foi realizado utilizando os testes de Mann-Whitney e/ou Kruskal-Wallis. A correlação de Spearman foi realizada a fim de verificar a relação dos sintomas osteomusculares com a qualidade de vida. Em todas as análises foi adotado um nível de significância de $5 \%(p<0,05)$.

O estudo foi aprovado pelo Comitê de Ética em Pesquisa (CEP) da Pontifícia Universidade Católica de Goiás (PUC Goiás), sob o parecer 꾸⒈875.687, CAAE: 62219516.8 .0000 .0037 . Dessa forma todos os participantes assinaram ao Termo de Consentimento Livre e Esclarecido.

\section{Resultados}

Foram pesquisados 52 professores, sendo $96,2 \%$ do sexo feminino e média de idade de $47,8( \pm 6,1)$ anos, e $59,6 \%$ tinham menos de 50 anos. A renda bruta mensal para $30,8 \%$ foi de um a três salários mínimos e para $69,2 \%$ foi de quatro a seis salários mínimos. A maioria dos professores eram casados $(69,2 \%)$ e tinham filhos $(86,5 \%)$.

Todos os professores possuíam mais de 10 anos de trabalho em regime estatutário no município de Guanambi/BA, com 92,3\% possuindo pós-graduação e $7,7 \%$ com ensino superior completo, com a maioria $(88,5 \%)$ com jornada de trabalho de 40 horas semanais.

Quanto à satisfação no trabalho $86,5 \%$ disseram estar satisfeitos no trabalho. No entanto $96,2 \%$ entenderam que seu trabalho tem risco à saúde e $96,2 \%$ consideraram seu ambiente de trabalho bom. Um total de $65,4 \%$ afirmou que se cansa com frequência. A grande maioria $(94,2 \%)$ usava computador, praticava atividade física $(55,8 \%)$ e realizava tarefas domésticas $(96,2 \%)$.

Dos 52 indivíduos, um total de $84,6 \%$ (44 indivíduos) apresentou queixa em uma ou mais de uma parte do corpo e 15,4\% (8 indivíduos) do total de participantes 
As quatro partes do corpo mais prevalentes na identificação de queixas nos últimos 12 meses anteriores a pesquisa foram: joelho (38,5\%), lombar $(34,6 \%)$, ombros $(34,6 \%)$ e pescoço $(32,7 \%)$ (Tabela 2). E nos últimos 7 dias anteriores a pesquisa foram: ombros $(26,8 \%)$, lombar $(25 \%)$, joelho $(23,1 \%)$ e pescoço $(23,1 \%)$. Os afastamentos mais frequentes do trabalho relacionados às queixas de sintomas osteomusculares foram: joelho $(17,3 \%)$, ombros $(17,3 \%)$, lombar $(15,4 \%)$ e pescoço $(11,5 \%)$ (Tabela 1).

Quanto a média da escala de dor referidas pelo conjunto dos professores pesquisados em relação as queixas, em primeiro lugar ficou a média de dor da região lombar $(5,8 \pm 1,7)$, seguida de dor nos ombros $(5,7 \pm 2,4)$, punhos/mãos $(5,7 \pm 2,6)$ e pescoço $(5,2 \pm 2,4)$ (Tabela 1$)$.

Tabela 1. Descrição dos sintomas osteomusculares (frequência) e média da dor dos 52 professores do ensino fundamental, Guanambi/BA, 2018.

\begin{tabular}{lcccc}
\hline & $\mathbf{1 2}$ meses & $\mathbf{7}$ meses & Afastamento & $\begin{array}{c}\text { Dor * } \\
\mathrm{n}(\%)\end{array}$ \\
$\mathrm{n}(\%)$ & $\mathrm{n}(\%)$ & Média \pm Desvio padrão \\
\hline Joelhos & $20(38,5)$ & $12(23,1)$ & $09(17,3)$ & $4,7 \pm 2,6$ \\
Ombros & $18(34,6)$ & $14(26,9)$ & $09(17,3)$ & $5,7 \pm 2,4$ \\
Lombar & $18(34,6)$ & $13(25,0)$ & $08(15,4)$ & $5,8 \pm 1,7$ \\
Pescoço & $17(32,7)$ & $12(23,1)$ & $06(11,5)$ & $5,2 \pm 2,4$ \\
Punho/Mãos & $12(23,1)$ & $08(15,4)$ & $05(9,6)$ & $5,7 \pm 2,6$ \\
Torácica & $10(19,2)$ & $03(5,8)$ & $05(9,6)$ & $4,0 \pm 1,7$ \\
Tornozelo/Pés & $07(13,5)$ & $05(9,6)$ & $04(7,7)$ & $3,7 \pm 2,0$ \\
Cotovelo & $04(7,7)$ & $02(3,8)$ & $02(3,8)$ & $4,3 \pm 2,9$ \\
Ancas/Coxas & $04(7,7)$ & $02(3,8)$ & $03(5,8)$ & $4,5 \pm 2,7$ \\
\hline
\end{tabular}

${ }^{*}$ Respostas negativas foram omitidas nas médias de dor.

Fonte: Dados da pesquisa.

$\mathrm{Na}$ análise estatística dos sintomas osteomusculares em relação ao perfil sociodemográfico ocupacional houve relação significativa entre 0 afastamento e 0 nível de escolaridade $(p=0,04)$, onde indivíduos que possuíam pós-graduação apresentaram menor escore de afastamento do que os que tinham ensino superior. Outro achado foi em relação ao afastamento e se sentir cansado no trabalho ( $p=$ 0,04 ), evidenciando que quanto mais o sentimento de cansaço maior foi o escore de afastamento por problemas osteomusculares (Tabela 2). 


\section{Revista Labor}

Programa de Pós-graduação em Educação, Universidade Federal do Ceará

Fortaleza-CE-Brasil

Tabela 2. Comparação dos sintomas osteomusculares com o perfil sociodemográfico ocupacional dos 52 professores do ensino fundamental, Guanambi/BA, 2018.

\begin{tabular}{|c|c|c|c|c|}
\hline & \multicolumn{4}{|c|}{ Sintomas osteomusculares (Média \pm DP) } \\
\hline & 12 meses & 7 dias & Afastamento & Dor \\
\hline \multicolumn{5}{|l|}{ Faixa etária* } \\
\hline \multicolumn{5}{|l|}{$\begin{array}{l}<50 \text { anos } \\
\geq 50 \text { anos }\end{array}$} \\
\hline$\geq 50$ anos & $11,6 \pm 2,4$ & $10,9 \pm 2,3$ & $10,3 \pm 1,9$ & $5,7 \pm 1,9$ \\
\hline \multicolumn{5}{|l|}{$\begin{array}{l}p \text { valor } \\
\text { Gênero* }\end{array}$} \\
\hline Feminino & $11,8 \pm 2,6$ & $10,8 \pm 2,2$ & $10.3 \pm 2.0$ & $5.0 \pm 2.0$ \\
\hline Masculino & $12.5 \pm 3.5$ & $12,0 \pm 4.2$ & $9.5 \pm 0.7$ & $7.0 \pm 2.8$ \\
\hline \multirow{2}{*}{\multicolumn{5}{|c|}{$\begin{array}{l}\text { p valor } \\
\text { Renda mensal* }\end{array}$}} \\
\hline & $12.6 \pm 3.0$ & $11.3 \pm 2.9$ & $11,2 \pm 2.9$ & $5,6 \pm 2,4$ \\
\hline 4 a 6 & $11,5 \pm 2,4$ & $10,7 \pm 1,9$ & $9,9 \pm 1,2$ & $4,8 \pm 1.8$ \\
\hline p valor & $0, \overline{1} 7$ & $0, \overline{89}$ & 0,24 & 0,17 \\
\hline \multicolumn{5}{|l|}{ Estado civil ${ }^{\star *}$} \\
\hline Separado & $\begin{array}{l}11,9 \pm 2,6 \\
11,0 \pm 2,4\end{array}$ & $\begin{array}{l}10,8 \pm 2,3 \\
10.8 \pm 2,5\end{array}$ & $10,2 \pm 1,8$ & $5,3 \pm 2,1$ \\
\hline $\begin{array}{l}\text { Solteiro } \\
\text { Soin }\end{array}$ & $12,1 \pm 3,1$ & $11,1 \pm 2,4$ & $10,7 \pm 3,0$ & $4.4 \pm 2.1$ \\
\hline Viúvo & $11.0 \pm 1.4$ & $11.0 \pm 1.4$ & $11.0 \pm 1.4$ & $5.0 \pm 0.0$ \\
\hline \multicolumn{5}{|l|}{$\begin{array}{l}p \text { valor } \\
\text { Tem filhos* }\end{array}$} \\
\hline Não & $11,4 \pm 2,0$ & $10,6 \pm 1,7$ & $9,9 \pm 1,2$ & $3.9 \pm 2.3$ \\
\hline Sim & $11,9 \pm 2,7$ & $10,9 \pm 2,3$ & $10,4 \pm 2,0$ & $5,3 \pm 2,0$ \\
\hline \multicolumn{5}{|l|}{ Escolaridade* } \\
\hline $\begin{array}{l}\text { Escolaridade } \\
\text { Ensino superior }\end{array}$ & $14.0+4.4$ & $13,0 \pm 3,2$ & $13.8+4.0$ & $5.2+0.5$ \\
\hline Pós-araduacão & $11,6 \pm 2.4$ & $10.7 \pm 2.1$ & $10.0 \pm 1,4$ & $5,1 \pm 2,1$ \\
\hline \multicolumn{5}{|l|}{$\begin{array}{l}\text { o valor } \\
\text { Jornada semanal* }\end{array}$} \\
\hline & $10,8 \pm 2,3$ & $10,7 \pm 2,4$ & $9,5 \pm 1,2$ & $4,3 \pm 1,5$ \\
\hline 40 horas & $11,9 \pm 2,6$ & $10,9 \pm 2,3$ & $10,4 \pm 2,0$ & $5,2 \pm 2,1$ \\
\hline p valor & 0,26 & 0,69 & 0,14 & \\
\hline Risco à saúde* & & & & \\
\hline $\begin{array}{l}\text { Não } \\
\text { Sim }\end{array}$ & $11,8 \pm 2,6$ & $10,9 \pm 2,3$ & $10,4 \pm 2,0$ & $5,0 \pm 2,0$ \\
\hline Sim & $12.5 \pm 5.0$ & $10.5 \pm 2.1$ & $9.0 \pm 0.0$ & $6.7 \pm 0.0$ \\
\hline \multicolumn{5}{|l|}{$\begin{array}{l}\text { D valor } \\
\text { Satisfacão no trabalho** }\end{array}$} \\
\hline Insatisfeito & $11,7 \pm 2,9$ & $11,3 \pm 3,2$ & $10,3 \pm 0,6$ & $4,1 \pm 1,0$ \\
\hline Muito satisfeito & $12,0 \pm 2,9$ & $12,0 \pm 2,9$ & $11,3 \pm 2,9$ & $5,9 \pm 1,5$ \\
\hline Satisfeito & $11,8 \pm 2,6$ & $10,8 \pm 2,1$ & $10,2 \pm 2,0$ & $5,1 \pm 2,1$ \\
\hline \multicolumn{5}{|l|}{$\begin{array}{l}\text { p valor } \\
\text { Ambiente de trabalho* }\end{array}$} \\
\hline Bom & $11.9 \pm 2.6$ & $10.9 \pm 2.3$ & $10.3 \pm 2.0$ & $5.1 \pm 1.9$ \\
\hline Excelente & $10,0 \pm 0,0$ & $9,5 \pm 0,7$ & $10,0 \pm 0,0$ & $5,0 \pm 5,7$ \\
\hline p valor & 0.33 & 0.44 & 0.57 & 0.98 \\
\hline Cansaco* & $11,4 \pm 2.3$ & $102+18$ & $96+0.9$ & $50+23$ \\
\hline $\mathrm{Sim}$ & $12,0 \pm 2,8$ & $11,3 \pm 2,4$ & $10,7 \pm 2,2$ & $5,1 \pm 1.9$ \\
\hline \multirow{2}{*}{\multicolumn{5}{|c|}{$\begin{array}{l}\text { p valor } \\
\text { Utiliza o computador* }\end{array}$}} \\
\hline & & & & \\
\hline Não & $9.7 \pm 0.6$ & $9.3 \pm 0.6$ & $9.7 \pm 0.6$ & $4.0 \pm 1.4$ \\
\hline Sim & $11,9 \pm 2,6$ & $11,0 \pm 2,3$ & $10,4 \pm 2,0$ & $5,1 \pm 2,1$ \\
\hline \multirow{2}{*}{\multicolumn{5}{|c|}{ Atividade física* }} \\
\hline & & & $107+23$ & $52+20$ \\
\hline & $11,5 \pm 2,8$ & $10,5 \pm 2,1$ & $10,0 \pm 1,7$ & $49+2.1$ \\
\hline$p$ valor & 0.12 & 0.22 & $0 . \overline{2} 8$ & 0.57 \\
\hline
\end{tabular}

*Mann-Whitney; **Kruskal-Wallis

Fonte: Dados da pesquisa.

No conjunto dos 52 professores pesquisados, a qualidade de vida total, através dos dados do WHOQOL-Bref, apresentou média de 3,6 $( \pm 0,3)$. A percepção 


\section{Revista Labor}

Programa de Pós-graduação em Educação, Universidade Federal do Ceará

Fortaleza-CE-Brasil

da qualidade de vida apresentou um escore de $3,8( \pm 0,4)$ e a satisfação pessoal com a própria saúde foi de $3,5( \pm 0,8)$ (Tabela 3$)$.

Ao analisar cada domínio separadamente, estes apresentaram escores médios indicando boa (relações sociais e meio ambiente) e muito boa (físico e psicológico) qualidade de vida, como seguem os resultados de cada domínio: Físico $(4,4 \pm 0,6)$, Psicológico $(4,7 \pm 0,4)$, Relações sociais $(3,8 \pm 0,6)$ e Meio ambiente $(3,7 \pm 0,4)$ (Tabela 3).

Tabela 3. Análise da qualidade de vida medida pelo WHOQOL-Bref dos 52 professores do ensino fundamental, Guanambi/BA, 2018.

\begin{tabular}{lccc}
\hline & Mediana & Média & Desvio Padrão \\
\hline Percepção da QV & 4,0 & 3,8 & 0,4 \\
Satisfação com a QV & 4,0 & 3,5 & 0,8 \\
Escore total & 3,7 & 3,6 & 0,3 \\
Físico & 4,5 & 4,4 & 0,6 \\
Psicológico & 4,8 & 4,7 & 0,4 \\
Social & 4,0 & 3,8 & 0,6 \\
Ambiental & 3,7 & 3,7 & 0,4 \\
\hline
\end{tabular}

Fonte: Dados da pesquisa.

Ao relacionar os aspectos da qualidade de vida com o perfil sociodemográfico ocupacional, identificou-se, com significância estatística, que a satisfação com a qualidade de vida foi maior nos professores da faixa etária abaixo dos 50 anos $(p=0,04)$, naqueles que referiram não se cansar no ambiente de trabalho $(p<0,001)$ e nos que utilizavam regularmente o computador para as funções laborais $(p=0,02)($ Tabela 4$)$.

O escore total de qualidade de vida apresentou melhor escore, nos que afirmaram receber de quatro a seis salários mínimos $(p=0,03)$, para os que entendem que seu ambiente laboral possui riscos à saúde $(p=0,03)$, naqueles que referiram não se cansar no ambiente de trabalho $(p<0,001)$ e nos que praticavam atividade física $(p=0,02)$ (Tabela 4). 
Tabela 4. Comparação dos aspectos da qualidade de vida com o perfil sociodemográfico ocupacional dos 52 professores do ensino fundamental, Guanambi/BA, 2018.

\section{Aspectos da qualidade de vida (Média \pm DP)}

\begin{tabular}{|c|c|c|c|}
\hline & Percepção da QV & Satisfação com a QV & Escore total \\
\hline \multicolumn{4}{|l|}{ Faixa etária* } \\
\hline$<50$ anos & $3.8 \pm 0.4$ & $3.7 \pm 0.7$ & $3.7 \pm 0.3$ \\
\hline$\geq 50$ anos & $3,7 \pm 0,5$ & $3,2 \pm 0,9$ & $3,6 \pm 0,3$ \\
\hline \multicolumn{4}{|l|}{ Gênero* } \\
\hline Feminino & $3.7 \pm 0.4$ & $3,5 \pm 0.8$ & $36+03$ \\
\hline Masculino & $4,0 \pm 0,0$ & $4,0 \pm 0,0$ & $3,8 \pm 0,1$ \\
\hline \multirow{2}{*}{\multicolumn{4}{|c|}{ Renda mensal* }} \\
\hline & & & \\
\hline 1 a 3 & $3.6 \pm 0.5$ & $3.4 \pm 1.0$ & $3.5 \pm 0.3$ \\
\hline 4 a 6 & $3,8 \pm 0,4$ & $3,5 \pm 0,7$ & $3,7 \pm 0,3$ \\
\hline o valor & 0.17 & 0.64 & 0.03 \\
\hline \multicolumn{4}{|l|}{ Estado civil ${ }^{\star \star}$} \\
\hline Separado & $3.8+0.5$ & $3.6 \pm 0.1$ & $\begin{array}{l}3.6 \pm 0.3 \\
36+0.3\end{array}$ \\
\hline Solteiro & $3,7 \pm 0,5$ & $3,4 \pm 1,0$ & $3,6 \pm 0,3$ \\
\hline Viúvo & $3,5 \pm 0,7$ & $3,0 \pm 0,0$ & $3,8 \pm 0,0$ \\
\hline p valor & 0,76 & 0,52 & 0,90 \\
\hline \multicolumn{4}{|l|}{ Tem filhos* } \\
\hline Não & $3.6 \pm 0.5$ & $3.6 \pm 1.0$ & $3.7 \pm 0.3$ \\
\hline Sim & $3,8 \pm 0,4$ & $3,5 \pm 0,8$ & $3,6 \pm 0,3$ \\
\hline \multicolumn{4}{|l|}{$\begin{array}{l}\text { o valor } \\
\text { Escolaridade* }\end{array}$} \\
\hline $\begin{array}{l}\text { Escolaridade } \\
\text { Ensino superior }\end{array}$ & $3.5 \pm 0.6$ & $3.5 \pm 1.0$ & $3,4 \pm 0,4$ \\
\hline Pós-araduação & $3,8 \pm 0,4$ & $3,5 \pm 0,8$ & $3,7 \pm 0,3$ \\
\hline \multirow{2}{*}{\multicolumn{4}{|c|}{$\begin{array}{l}p \text { valor } \\
\text { Jornada semanal* }\end{array}$}} \\
\hline & & & \\
\hline 40 horas & $3,8 \pm 0,4$ & $\begin{array}{l}3,0 \pm 0,8 \\
3,4 \pm 0,8\end{array}$ & $\begin{array}{l}3.1 \pm 0.2 \\
3.6 \pm 0.3\end{array}$ \\
\hline p valor & $0 . \overline{6} 2$ & 0.33 & 0.92 \\
\hline \multicolumn{4}{|l|}{ Risco à saúde* } \\
\hline Não & $3,7 \pm 0,4$ & $3,5 \pm 0,8$ & $3,6 \pm 0,3$ \\
\hline Sim & $4,0 \pm 0,0$ & $4,0 \pm 0,0$ & $4,0 \pm 0,0$ \\
\hline \multirow{2}{*}{\multicolumn{4}{|c|}{ Satisfacão no trabalho** }} \\
\hline & & & \\
\hline Insatisfeito & $3,7 \pm 0,6$ & $2.3 \pm 0.6 \mathbf{b}$ & $3.3 \pm 0.1$ \\
\hline Muito satisfeito & $3,8 \pm 0,5$ & $3,5 \pm 1,0 \mathbf{a}$ & $3,7 \pm 0,5$ \\
\hline Satisfeito & $3.8 \pm 0.4$ & $3.6 \pm 0.8 \mathbf{a}$ & $3.7 \pm 0.3$ \\
\hline \multicolumn{4}{|l|}{ Ambiente de trabalho* } \\
\hline $\begin{array}{l}\text { Amplente de trabaino" } \\
\text { Bom }\end{array}$ & $3.7+0.4$ & $3.5+0.8$ & $3.6+0.3$ \\
\hline Excelente & $4.0 \pm 0.0$ & $4.0 \pm 0.0$ & $3.9 \pm 0.0$ \\
\hline$p$ valor & 0,41 & 0,31 & 0,23 \\
\hline \multicolumn{4}{|l|}{ Cansaco* } \\
\hline Não & $3,8 \pm 0,4$ & $3,9 \pm 0,6$ & $3,8 \pm 0,2$ \\
\hline Sim & $3.7 \pm 0.5$ & $3.2 \pm 0.8$ & $3.6 \pm 0.3$ \\
\hline \multirow{2}{*}{\multicolumn{4}{|c|}{$\begin{array}{l}\text { p valor } \\
\text { Utiliza o computador* }\end{array}$}} \\
\hline & & & \\
\hline Não & $3,7 \pm 0,6$ & $2,3 \pm 0,6$ & $3,4 \pm 0,1$ \\
\hline Sim & $3,8 \pm 0,4$ & $3,6 \pm 0,8$ & $3,7 \pm 0,3$ \\
\hline \multirow{2}{*}{\multicolumn{4}{|c|}{ Atividade física* }} \\
\hline & & & \\
\hline $\begin{array}{l}\text { Não } \\
\text { Sim }\end{array}$ & $\begin{array}{l}3,7 \pm 0,5 \\
3,8 \pm 0,4\end{array}$ & $\begin{array}{l}3,4 \pm 0,1 \\
3,6 \pm 0,9\end{array}$ & $\begin{array}{l}3,6 \pm 0,3 \\
37+0,3\end{array}$ \\
\hline $\begin{array}{l}\text { Sim } \\
\text { p valor }\end{array}$ & $\begin{array}{c}3.8 \pm 0.4 \\
0,42\end{array}$ & $\begin{array}{c}0,0 \pm 0.9 \\
0,37\end{array}$ & 0,02 \\
\hline
\end{tabular}

*Mann-Whitney; **Kruskal-Wallis

Fonte: Dados da pesquisa. 
Na relação entre os domínios do WHOQOL-Bref e o perfil sociodemográfico ocupacional, o domínio meio ambiente apresentou significância estatística com melhor escore para os indivíduos que afirmaram receber uma renda mensal de quatro a seis salários mínimos $(p=0,03)$ e com maior satisfação do trabalho $(p=0,04)$. Os professores que referiram não se cansarem obtiveram melhor escore de qualidade de vida nos domínios físico $(p=0,01)$, social $(p=0,01)$ e ambiental $(p=0,01)$ (Tabela 5).

Tabela 5. Comparação dos domínios do WHOQOL-Bref com o perfil sociodemográfico ocupacional dos 52 professores do ensino fundamental, Guanambi/BA, 2018.

\begin{tabular}{|c|c|c|c|c|}
\hline & \multicolumn{4}{|c|}{ Domínios WHOQOL-Bref (Média \pm DP) } \\
\hline & Físico & Psicológico & Social & Ambiental \\
\hline $\begin{array}{l}\text { Faixa etária* } \\
<50 \text { anos } \\
\geq 50 \text { anos } \\
0 \text { valor }\end{array}$ & $\begin{array}{l}4.4 \pm 0.6 \\
4,4 \pm 0,5 \\
0.93\end{array}$ & $\begin{array}{c}4,8 \pm 0.4 \\
4,6 \pm 0.4 \\
0.11\end{array}$ & $\begin{array}{c}3,8 \pm 0.7 \\
3,7 \pm 0.4 \\
0.38\end{array}$ & $\begin{array}{c}3.8 \pm 0.3 \\
3.6 \pm 0.4 \\
0.07\end{array}$ \\
\hline $\begin{array}{l}\text { Gênero* } \\
\text { Feminino } \\
\text { Masculino } \\
\text { p valor } \\
\text { Renda mensal* }\end{array}$ & $\begin{array}{c}4,4 \pm 0,6 \\
4,9 \pm 0,2 \\
0,20\end{array}$ & $\begin{array}{l}4,7 \pm 0,4 \\
5,1 \pm 0,4 \\
\quad 0,23\end{array}$ & $\begin{array}{c}3,8 \pm 0,6 \\
3,7 \pm 0,0 \\
0,52\end{array}$ & $\begin{array}{c}3,7 \pm 0,4 \\
3,7 \pm 0,3 \\
0,72\end{array}$ \\
\hline $\begin{array}{l}\text { Renar } \\
1 \text { a } 3 \\
4 \text { a } 6 \\
\text { p valor } \\
\text { Estado civil }\end{array}$ & $\begin{array}{l}4.2 \pm 0.7 \\
4,5 \pm 0.5 \\
0.16\end{array}$ & $\begin{array}{l}4.6 \pm 0.5 \\
4,8 \pm 0.4 \\
0.27\end{array}$ & $\begin{array}{l}3.6 \pm 0.7 \\
3,9 \pm 0.5 \\
0.33\end{array}$ & $\begin{array}{c}3.8 \pm 0.3 \\
3,8 \pm 0.4 \\
\quad 0.03\end{array}$ \\
\hline $\begin{array}{l}\text { Casado } \\
\text { Separado } \\
\text { Solteiro } \\
\text { Viúvo } \\
p \text { valor } \\
\text { Tem filhos* }\end{array}$ & $\begin{array}{l}4,4 \pm 0,5 \\
4,5 \pm 0,6 \\
4,4 \pm 0,7 \\
4,8 \pm 0,2 \\
0.62\end{array}$ & $\begin{array}{l}4,7 \pm 0,4 \\
4,8 \pm 0,4 \\
4,8 \pm 0.5 \\
5,0 \pm 0,0 \\
0.65\end{array}$ & $\begin{array}{c}3,9 \pm 0,5 \\
3,5 \pm 0,5 \\
3,6 \pm 0.9 \\
3,5 \pm 0,7 \\
0.40\end{array}$ & $\begin{array}{c}3,7 \pm 0,4 \\
3,6 \pm 0,4 \\
3,8 \pm 0,4 \\
3,8 \pm 0,3 \\
0.83\end{array}$ \\
\hline $\begin{array}{l}\text { Não } \\
\text { Sim } \\
\text { p valor } \\
\text { Escolaridade* }\end{array}$ & $\begin{array}{c}4,6 \pm 0.5 \\
4,4 \pm 0,6 \\
0,22\end{array}$ & $\begin{array}{c}4.9 \pm 0.5 \\
4,7 \pm 0.4 \\
0,17\end{array}$ & $\begin{array}{c}3,7 \pm 0.6 \\
3,8 \pm 0,6 \\
0,83\end{array}$ & $\begin{array}{l}3.8 \pm 0.4 \\
3,7 \pm 0,4 \\
\quad 0,56\end{array}$ \\
\hline $\begin{array}{l}\text { Ensino superior } \\
\text { Pós-araduação } \\
\text { o valor }\end{array}$ & $\begin{array}{l}3,8 \pm 0,9 \\
4,5 \pm 0,5 \\
0,12\end{array}$ & $\begin{array}{c}4,5 \pm 0,6 \\
4,8 \pm 0,4 \\
0.38\end{array}$ & $\begin{array}{c}3,4 \pm 1,0 \\
3,8 \pm 0,5 \\
0,58\end{array}$ & $\begin{array}{l}3,7 \pm 0,2 \\
3,7 \pm 0,4 \\
\quad 0,85\end{array}$ \\
\hline $\begin{array}{l}20 \text { horas } \\
40 \text { horas } \\
\text { p valor } \\
\text { Risco à saúde* }\end{array}$ & $\begin{array}{l}4,6 \pm 0.3 \\
4,4 \pm 0,6 \\
0,40\end{array}$ & $\begin{array}{l}4,8 \pm 0.5 \\
4,7 \pm 0,4 \\
0,45\end{array}$ & $\begin{array}{c}3,6 \pm 0.5 \\
3,8 \pm 0,6 \\
0,24\end{array}$ & $\begin{array}{c}3,7 \pm 0,2 \\
3,7 \pm 0,4 \\
0,90\end{array}$ \\
\hline $\begin{array}{l}\text { Não } \\
\text { Sim } \\
\text { D valor } \\
\text { Satisfacão no trabalho** }\end{array}$ & $\begin{array}{c}4,4 \pm 0,65 \\
5,0 \pm 0,2 \\
0,07\end{array}$ & $\begin{array}{l}4,7 \pm 0,4 \\
5,0 \pm 0,3 \\
0.35\end{array}$ & $\begin{array}{l}3,8 \pm 0,6 \\
4,0 \pm 0,0 \\
0,49\end{array}$ & $\begin{array}{l}3,7 \pm 0,4 \\
4,1 \pm 0,1 \\
\quad 0,10\end{array}$ \\
\hline $\begin{array}{l}\text { Insatisfeito } \\
\text { Muito satisfeito } \\
\text { Satisfeito } \\
\text { p valor } \\
\text { Ambiente de trabalho* }\end{array}$ & $\begin{array}{l}4,0 \pm 0.3 \\
4,5 \pm 1,1 \\
4,4 \pm 0.5 \\
\quad 0,13\end{array}$ & $\begin{array}{l}4.5 \pm 0.3 \\
5,1 \pm 0,2 \\
4,7 \pm 0.4 \\
\quad 0,12\end{array}$ & $\begin{array}{c}3,7 \pm 0.3 \\
3,3 \pm 0,8 \\
3,8 \pm 0.6 \\
0,42\end{array}$ & $\begin{array}{c}3,2 \pm 0,2 b \\
3,8 \pm 0,4 \mathbf{a} \\
3,7 \pm 0,3 \mathbf{a} \\
\mathbf{0 , 0 4}\end{array}$ \\
\hline $\begin{array}{l}\text { Bom } \\
\text { Excelente } \\
\text { p valor } \\
\text { Cansaco* }\end{array}$ & $\begin{array}{l}4,4 \pm 0,6 \\
4,7 \pm 0.5 \\
0,50\end{array}$ & $\begin{array}{l}4,7 \pm 0,4 \\
4,8 \pm 0.0 \\
0,89\end{array}$ & $\begin{array}{c}3,8 \pm 0,6 \\
4,2 \pm 0,7 \\
0,42\end{array}$ & $\begin{array}{c}3,7 \pm 0,4 \\
3.9 \pm 0.1 \\
0,29\end{array}$ \\
\hline Não & $4,7 \pm 0,4$ & $4,8 \pm 0,4$ & $4,1 \pm 0,4$ & $3,9 \pm 0,3$ \\
\hline
\end{tabular}

DOI: https://doi.org/10.29148/labor.v1i25.60024 
Programa de Pós-graduação em Educação, Universidade Federal do Ceará

Fortaleza-CE-Brasil

\begin{tabular}{|c|c|c|c|c|}
\hline $\begin{array}{l}\text { Sim } \\
\text { p valor }\end{array}$ & $\begin{array}{l}4,3 \pm 0,6 \\
0.01\end{array}$ & $\begin{array}{l}4,7 \pm 0,5 \\
0.59\end{array}$ & $\begin{array}{l}3,6 \pm 0,6 \\
0.01\end{array}$ & $\begin{array}{l}3,6 \pm 0,4 \\
0.01\end{array}$ \\
\hline Utiliza o computador ${ }^{\star}$ & & & & \\
\hline Não & $4.2 \pm 0.1$ & $4.5 \pm 0.2$ & $3.6 \pm 0.4$ & $3.4 \pm 0.1$ \\
\hline $\begin{array}{l}\text { Sim } \\
p \text { valor }\end{array}$ & $4,4 \pm 0,6$ & $4,8 \pm 0,4$ & $3,8 \pm 0,6$ & $3,7 \pm 0,4$ \\
\hline $\begin{array}{l}\text { p valor } \\
\text { Atividade física* }\end{array}$ & 0,25 & 0,27 & 0,33 & \\
\hline Não & $4,2 \pm 0.6$ & $4.6 \pm 0.4$ & $3.7 \pm 0.7$ & $3.7 \pm 0.4$ \\
\hline Sim & $4,5 \pm 0,5$ & $4,8 \pm 0,5$ & $3,9 \pm 0,5$ & $3,7 \pm 0,3$ \\
\hline o valor & 0.06 & 0.13 & 0.60 & 0.36 \\
\hline
\end{tabular}

*Mann-Whitney; **Kruskal-Wallis

Fonte: Dados da pesquisa.

$\mathrm{Na}$ correlação entre sintomas osteomusculares e a qualidade de vida, identificou-se que os professores com sintomas osteomusculares nos últimos 12 meses apresentaram escores inversamente proporcionais em relação aos escores de qualidade de vida total $(r=-0,40 ; p<0,001)$ e no domínio físico $(r=-0,49 ; p<0,001)$ (Tabela 6). Tais valores caracterizam uma relação negativa em que $o$ aumento dos sintomas osteomusculares decresce a qualidade de vida.

Os professores que referiram sintomas osteomusculares nos últimos 7 dias apresentaram escores inversamente proporcionais em relação à qualidade de vida total $(r=-0,42 ; p<0,001)$, no domínio físico $(r=-0,42 ; p<0,001)$, no domínio ambiental $(r=-0,32 ; p=0,02)$ e na satisfação com a qualidade de vida $(r=-0,36 ; p$ $=0,01$ ) (Tabela 6 ). A mesma associação negativa ocorre também nessa relação como na anterior.

Os professores que afirmaram ter tido afastamento do serviço por problemas osteomusculares apresentaram escores inversamente proporcionais quanto à percepção da própria qualidade de vida $(r=-0,31 ; p=0,03)$, na satisfação com a qualidade de vida $(r=-0,50 ; p<0,001)$, no escore total $(r=-0,51 ; p<0,001)$, bem como nos domínios físico $(r=-0,45 ; p<0,001)$, social $(r=-0,31 ; p=0,03)$ e ambiental $(r=-0,33 ; p=0,02)$ (Tabela 6$)$. Essa relação apresentou-se negativa em quase todos os escores, somente no domínio físico não houve significância. 
Programa de Pós-graduação em Educação, Universidade Federal do Ceará

Fortaleza-CE-Brasil

Tabela 6. Correlaçáo de Spearman entre a Qualidade de vida e os sintomas osteomusculares dos 52 professores do ensino fundamental, Guanambi/BA, 2018.

\section{Sintomas Osteomusculares}

\begin{tabular}{|c|c|c|c|c|}
\hline WHOQOL-Bref & 12 meses & 7 dias & Afastamento & Dor \\
\hline $\begin{array}{l}\text { Percepção da QV } \\
p \text { valor }\end{array}$ & $\begin{array}{c}r=-0,24 \\
0,09\end{array}$ & $\begin{array}{c}r=-0,20 \\
0,15\end{array}$ & $\begin{array}{c}r=-0,31 \\
0,03\end{array}$ & $\begin{array}{c}r=0,04 \\
0,80\end{array}$ \\
\hline $\begin{array}{l}\text { Satisfação com a QV } \\
p \text { valor }\end{array}$ & $\begin{array}{c}r=-0,23 \\
0,09\end{array}$ & $\begin{array}{c}r=-0,36 \\
0,01\end{array}$ & $\begin{array}{c}r=-0,50 \\
<0,001\end{array}$ & $\begin{array}{c}r=-0,09 \\
0,58\end{array}$ \\
\hline $\begin{array}{l}\text { Escore total } \\
p \text { valor }\end{array}$ & $\begin{array}{c}r=-0,40 \\
<0,001\end{array}$ & $\begin{array}{c}r=-0,42 \\
<0,001\end{array}$ & $\begin{array}{c}r=-0,51 \\
<0,001\end{array}$ & $\begin{array}{c}r=-0,05 \\
0,73\end{array}$ \\
\hline $\begin{array}{l}\text { Físico } \\
p \text { valor }\end{array}$ & $\begin{array}{c}r=-0,49 \\
<0,001\end{array}$ & $\begin{array}{c}r=-0,42 \\
<0,001\end{array}$ & $\begin{array}{c}r=-0,45 \\
<0,001\end{array}$ & $\begin{array}{c}r=0,05 \\
0,76\end{array}$ \\
\hline $\begin{array}{l}\text { Psicológico } \\
p \text { valor }\end{array}$ & $\begin{array}{c}r=-0,25 \\
0,07\end{array}$ & $\begin{array}{c}r=-0,12 \\
0,39\end{array}$ & $\begin{array}{c}r=-0,23 \\
0,10\end{array}$ & $\begin{array}{c}r=0,03 \\
0,86\end{array}$ \\
\hline $\begin{array}{l}\text { Social } \\
p \text { valor }\end{array}$ & $\begin{array}{c}r=-0,16 \\
0,24\end{array}$ & $\begin{array}{c}r=-0,18 \\
0,20\end{array}$ & $\begin{array}{c}r=-0,31 \\
0,03\end{array}$ & $\begin{array}{c}r=-0,08 \\
0,61\end{array}$ \\
\hline $\begin{array}{l}\text { Ambiental } \\
p \text { valor }\end{array}$ & $\begin{array}{c}r=-0,20 \\
0,15\end{array}$ & $\begin{array}{c}r=-0,32 \\
0,02\end{array}$ & $\begin{array}{c}r=-0,33 \\
0,02\end{array}$ & $\begin{array}{c}r=-0,02 \\
0,89\end{array}$ \\
\hline
\end{tabular}

Fonte: Dados da pesquisa.

\section{Discussão}

A presença de mulheres no ofício de professor no ensino fundamental parece ser preponderante. O Instituto de Pesquisa Econômica Aplicada (IPEA) apresentou dados de 2015 que identificaram uma grande representação das mulheres na condição de professoras da educação básica (IPEA, 2017). Outros estudos também confirmaram essa predominância (FERREIRA et al, 2015; MOREIRA; SANTINO; TOMAZ, 2017; PIMENTEL et al, 2016; ROCHA et al, 2017). Tal dado corrobora com a predominância de $96,2 \%$ do sexo feminino encontrado no presente estudo.

Quanto à faixa etária deste estudo, cuja média foi de 47,8 anos, assemelhase a vários estudos com professores do ensino fundamental e médio (FERREIRA et al., 2015; MOREIRA; SANTINO; TOMAZ, 2017; PIMENTEL et al., 2016). Porém os professores com menos de 10 anos de trabalho foram descartados desta pesquisa, 0 que pode ter aumentado a média de idade. No Censo escolar de 2016, a média de idade dos professores da educação básica foi de 40,1 anos (INEP, 2017), um pouco abaixo das médias deste estudo e dos estudos encontrados. 

básica possuem nível superior completo (INEP, 2017). Diverge deste estudo que apresentou em sua maioria o nível de pós-graduação (especialização). Os professores do município de Guanambi buscam continuidade de sua formação profissional, por motivos desconhecidos desta pesquisa.

Identificou-se uma maioria dos professores que relataram se cansar muito no ambiente de trabalho e que esse mesmo ambiente organizacional traz riscos a saúde. A percepção de cansaço e de risco à saúde pode estar relacionada com elevada carga horária de trabalho, número excessivo de turmas e de alunos, e pouco tempo para a organização das aulas. Também pode estar associada à presença de estresse relacionado ao trabalho, diminuindo, assim, o tempo de recuperação de energia física e mental (MOREIRA; SANTINO; TOMAZ, 2017).

No presente estudo a maioria $(55,8 \%)$ praticava atividade física, assim como em outro estudo com 126 professores do ensino fundamental que também apresentou alta prevalência de sintomas osteomusculares em que $55,5 \%$ praticavam exercícios como caminhada, ciclismo, musculação e dança (MANGO et al, 2012). Contrariando a tendência nacional de $37,9 \%$ e no Nordeste de $36,3 \%$. Na Bahia $37,1 \%$ praticavam algum esporte ou atividade física, entre setembro de 2014 e setembro de 2015 (na faixa etária de 15 anos ou mais) (IBGE, 2017b).

Entretanto, em outra pesquisa com 23 professores, em Campina Grande/PB, foi encontrado uma porcentagem de apenas $39,1 \%$ que praticavam algum tipo de atividade física, mas também alta prevalência de sintomas osteomusculares e depreciação da qualidade de vida (MOREIRA; SANTINO; TOMAZ, 2017).

Considerando que a atividade física é um fator positivo na qualidade de vida e na prevenção de doenças osteomusculares (AZEVEDO et al, 2016), deve-se ponderar os resultados em que os estudos apresentam alta prevalência de sintomas osteomusculares, porém com alta prática de atividade física, posto que no presente estudo não foi pesquisado o tipo de atividade física, há quanto tempo se realiza essas atividades e a frequência com a qual se realiza. Há que se investigar isso em estudos mais aprofundados e específicos.

Com relação aos sintomas osteomusculares, assim como no presente estudo, o perfil sociodemográfico dos trabalhadores notificados com LER/DORT no 


\section{Revista Labor}

Programa de Pós-graduação em Educação, Universidade Federal do Ceará

Fortaleza-CE-Brasil

Brasil e na Bahia, entre os anos de 2007 e 2012, foi semelhante com predominância do sexo feminino (52,6\% no Brasil e $51,5 \%$ na Bahia) entre as notificações (MELO et al., 2015).

A alta prevalência de sintomas osteomusculares encontrada neste estudo $(84,6 \%)$ também foi identificada em outros estudos como na pesquisa com professores $(60,5 \%)$ do ensino médio de quatro escolas de Ceilândia-DF (CALIXTO et al, 2015). Também na pesquisa com professores do ensino fundamental na cidade de Campina Grande/PB (82,6\%) (MOREIRA; SANTINO; TOMAZ, 2017). E em estudos internacionais, como por exemplo, com professores de Mugla/Turquia (77\%) (KARAKAYA et al, 2015) e de Botswana (83,3\%) (ERICK; SMITH, 2014).

O presente estudo apresentou maior prevalência de sintomas osteomusculares em quatro partes do corpo: joelho, ombro, pescoço e coluna lombar. Semelhanças foram encontradas no estudo de Calixto et al. (2015) em que as regiões corporais mais acometidas foram a parte superior das costas $(42,6 \%)$, a parte inferior das costas $(41,7 \%)$, o pescoço (39.3\%) e o ombro (30,4\%). No estudo que investigou a dor osteomuscular e seu impacto na qualidade de vida em professores da cidade de Mugla/Turquia identificou-se que as regiões dolorosas mais comuns foram o pescoço (39\%) e a parte inferior das costas (38\%) (KARAKAYA et al., 2015). Um outro estudo os distúrbios osteomusculares foram mais comuns em costas $(52,6 \%)$, ombros $(52,5 \%)$ e pescoço $(50,8 \%)$ (ERICK; SMITH, 2014).

A coluna também teve destaque em outro estudo ao considerar os sintomas relatados nos últimos 12 meses (47,8\%). Já quando se refere aos últimos sete dias um maior comprometimento de dor musculoesquelética foram referidas aos membros inferiores (34,8\%) (MOREIRA; SANTINO; TOMAZ, 2017).

É importante ressaltar que a dor persistente caracteriza cronicidade e acarreta adaptações do sistema osteomuscular e psicocomportamentais que podem interferir na capacidade funcional tanto nas atividades laborais quanto para atividades de vida diária (CALIXTO et al., 2015).

No presente estudo também houve mais afastamentos por queixas em ombros (17,3\%), joelhos (17,3\%), coluna lombar $(15,4 \%)$ e pescoço $(11,5 \%)$. Em relação ao afastamento de suas atividades normais de trabalho nos últimos 12 meses, os docentes de outro estudo referiram maior ocorrência de problemas na região da coluna $(34,8 \%)$, nos membros superiores $(34,8 \%)$, e nos membros inferiores $(34,8 \%)$ 
Em uma pesquisa sobre a distribuição da casuística de distúrbios osteomusculares relacionados ao trabalho (LER/DORT), com os dados do Brasil e da Bahia, 80,2\% das notificações nacionais sinalizaram afastamento das atividades; a Bahia também apresentou proporção elevada de afastamento, 75,2\% dos casos. Além disso, em ambos os contextos investigados, o desenvolvimento do distúrbio foi preponderante entre trabalhadores que referiram até 10 anos de tempo de trabalho na ocupação e o destaque da faixa etária ficou em torno de 36 a 50 anos (MELO et al., 2015). Já neste presente estudo os professores tinham mais de 10 anos de trabalho.

De acordo com a Pesquisa Nacional de Saúde do IBGE, no ano de 2013, a prevalência de DORT no Brasil teve maior proporção de diagnósticos no grupo de idade em que se concentram mais pessoas economicamente ativas: de 30 a 59 anos (3,2\%) (IBGE, 2014).

No presente estudo a faixa de idade foi de 36 a 61 anos e não houve relação significativa entre os sintomas osteomusculares e a faixa etária, porém, a satisfação com a QV foi maior nos professores da faixa etária abaixo de 50 anos. E o tempo de trabalho foi de mais de 10 anos. Logo percebe-se que quanto mais idade e tempo de trabalho mais chance de ter sintomas osteomusculares.

Em uma revisão de distúrbios musculoesqueléticos entre professores residentes em várias nações revelou-se que os professores da Turquia, China, Austrália, Brasil, Suécia, EUA, Alemanha, Estônia, Japão, Malásia, Filipinas, França e Grécia, demonstraram relação com outros grupos ocupacionais e uma alta prevalência de distúrbios musculoesqueléticos (MESARIA; JAISWAL, 2015).

Neste estudo, os escores demonstram boa qualidade de vida total, percepção da qualidade de vida e a satisfação pessoal. Já em outro estudo com professores, porém, em termos de percepção da qualidade de vida geral, os escores médios obtidos em dois estudos apontaram que há um índice considerável de professores com qualidade de vida geral abaixo do satisfatório (DAVOGLIO; LETTNIN; BALDISSERA, 2015).

Resultado inesperado ocorreu ao analisar cada domínio separadamente neste estudo, em que todos eles apresentaram escores médios indicando boa qualidade de vida com maior escore no domínio psicológico e o menor no domínio 


\section{Revista Labor}

Programa de Pós-graduação em Educação, Universidade Federal do Ceará

Fortaleza-CE-Brasil

ambiental. Diferentemente em uma revisão sistemática os resultados encontrados com o uso do WHOQOL-Bref em amostras do ensino básico, ficou evidenciado pequenas diferenças percentuais das médias em que o escore mais baixo foi no domínio ambiental e o mais elevado no domínio relações sociais (DAVOGLIO; LETTNIN; BALDISSERA, 2015).

Em outro estudo de qualidade de vida em docentes da área de saúde, o domínio físico se mostrou o mais afetado, pois apresentou menores valores médios ao passo que as relações sociais, com maior valor, foi o aspecto mais positivo da qualidade de vida dos docentes (MARQUES, 2016).

No presente estudo a satisfação com a qualidade de vida foi maior nos professores de faixa etária abaixo dos 50 anos. Diferença ocorreu no estudo de Marques (2016) em que houve associação estatisticamente significativa entre o domínio físico e a idade, com maiores escores para a faixa dos 44 anos ou mais.

A qualidade de vida geral, no presente estudo, apresentou melhor escore nos professores que afirmaram receber de quatro a seis salários mínimos, para os que apontaram que no seu ambiente laboral havia riscos à saúde, naqueles que referiram não se cansar no ambiente de trabalho e nos que praticavam atividade física. Os professores que referiram não se cansarem obtiveram melhor escore de qualidade de vida nos domínios físico, social e ambiental.

Em uma pesquisa com professores da educação básica do município de Florianópolis/SC, os professores apresentaram escores na avaliação da qualidade de vida que podem ser classificados como regulares. As variáveis mais associadas aos baixos escores na avaliação da qualidade de vida foram lecionar também na rede estadual, maior tempo de magistério e maior carga horária de trabalho semanal. Os domínios meio ambiente e físico apresentaram maior importância para a percepção geral de qualidade de vida (PEREIRA; TEIXEIRA; LOPES, 2013).

Identificou-se que os escores foram inversamente proporcionais quando relacionados os sintomas osteomusculares e em todos os aspectos relacionados à qualidade de vida. Percebe-se que a qualidade de vida total e o domínio físico foi afetado em todos resultados do questionário Nórdico. Sendo assim, nesta pesquisa, os sintomas osteomusculares apresentaram correlação negativa, demonstrando que se relacionam diretamente com a qualidade de vida dos professores do ensino fundamental do município de Guanambi/BA. 
Semelhante resultado foi encontrado no estudo que investigou professores do município de Campina Grande/PB quando comparou a qualidade de vida dos docentes com a prevalência de dor e necessidade de afastamento devido a dor com os que não relataram dores, em que houve depreciação da qualidade de vida dos docentes que relataram dor aguda, dos que relataram prevalência de dor crônica e dos que se afastaram das atividades por conta da presença de dor (MOREIRA; SANTINO; TOMAZ, 2017).

Outro estudo semelhante, na cidade de Mugla/Turquia, apresentou que os distúrbios osteomusculares tiveram impacto negativo, especialmente nos componentes físicos da qualidade de vida (KARAKAYA et al, 2015).

Em outro estudo foi evidenciado que os distúrbios osteomusculares dos professores reduziam as atividades em casa, enquanto alguns não conseguiam trabalhar por vários dias e outros precisavam procurar atendimento médico por causa da dor (ERICK; SMITH, 2014). Entretanto, já em uma pesquisa com professores do ensino básico de Caçador/SC, concluíram que a alta prevalência de sintomas osteomusculares e estresse não alteraram a qualidade de vida desses professores (ROCHA et al, 2017).

Considerando que os distúrbios osteomusculares são bastante expressivos nos estudos de saúde do professor, indicando alta prevalência em diferentes localidades de estudo e que também pode afetar a qualidade de vida, as investigações nesse âmbito podem contribuir para identificar os aspectos organizacionais da escola, os aspectos psicossociais e as demandas físicas enfrentadas pelos docentes em seu trabalho e como isso pode afetar sua vida.

As exigências de um mundo contemporâneo nas relações de trabalho tornam cada vez mais necessário compreender melhor o papel que o trabalho exerce na determinação do processo saúde-doença. Posto que os modelos tradicionais não atendem aos novos paradigmas na atual dinâmica, de um mundo diversificado, globalizado. Sendo necessária a investigação constante, análise e intervenção num processo contínuo de avaliação.

\section{Considerações Finais}

O perfil sociodemográfico dos professores apresentou prevalência de mulheres, em sua maioria com menos de 50 anos, casadas e com filhos. Todos 


\section{Revista Labor}

Programa de Pós-graduação em Educação, Universidade Federal do Ceará

Fortaleza-CE-Brasil

trabalhavam há mais de 10 anos em regime estatutário, com a maioria em jornada de 40 horas semanais possuindo nível superior e pós-graduação (especialização). Identificou-se, também, que a maioria relata se cansar muito e que o ambiente de trabalho trazia risco à saúde.

Quanto aos sintomas osteomusculares prevaleceu alta taxa de queixas $(86,4 \%)$ incluindo os sintomas para o último ano anterior à pesquisa, para os últimos sete dias anteriores e para os afastamentos. Em todos os casos as principais partes do corpo relacionadas às queixas foram os ombros, os joelhos, a coluna lombar e o pescoço. A média de dor ficou em 5,51 na escala de dor. $\mathrm{Na}$ análise entre sintomas osteomusculares e o perfil sociodemográfico houve evidência que quanto mais cansado no trabalho maior foi o afastamento por sintomas osteomusculares.

A qualidade de vida total, a percepção da qualidade de vida e a satisfação pessoal obtiveram boas médias apresentando o maior escore no domínio psicológico e o menor no domínio ambiental. Os professores que referiram não se cansar tiveram melhor escore de qualidade de vida nos domínios físico, social e ambiental.

Houve relação inversamente proporcional entre a qualidade de vida e os sintomas osteomusculares, somente o domínio psicológico não sofreu relação inversa, mas também não apresentou correlação significativa. Sendo que a qualidade de vida geral e o domínio físico foram associados de forma inversa em todos os resultados do questionário Nórdico. Esses resultados revelaram uma relação negativa entre os sintomas osteomusculares e a qualidade de vida.

Assim os dados nesta pesquisa possibilitaram evidenciar que os professores apresentaram alta prevalência de sintomas osteomusculares e que esses sintomas afetam diretamente a qualidade de vida dos professores do ensino fundamental do município de Guanambi/BA.

Assim, o conhecimento dos riscos e agravos à saúde relacionados ao trabalho é fundamental para o planejamento das ações de prevenção, promoção, assistência, vigilância e intervenção sobre os ambientes de trabalho, como também para a conduta dos trabalhadores e da sociedade.

\section{Agradecimentos}

Os autores gostariam de agradecer à Coordenação de Aperfeiçoamento de Pessoal de Nível Superior (CAPES) pela Bolsa de Mestrado concedida para a efetivação desta pesquisa. 


\section{Referências Bibliográficas}

ANDRADE, A. N. et al. Saúde na escola: o cuidado com professores. Revista Ciência e Extensão, v. 10, n. 1, p. 98-107, 2014.

AZEVEDO, E. M. Análise da qualidade de vida e do nível de atividade física dos policiais militares do comando geral da polícia miliar de Mato Grosso para prevenção de doenças. Revista de Administração do Sul do Pará, v. 3, n. 2, 2016.

BARROS, E. N. C.; ALEXANDRE, N. M. C. Cross-cultural adaptation of the Nordic musculoskeletal questionnaire. International Council of Nurses, International Nursing Review, v. 50, p. 101-108, 2003.

BUENO, C. O trabalho e o homem. 2015. Revista Pré Univesp. №. 59, Gênero, 2016. Disponível em: http://pre.univesp.br/o-trabalho-e-o-homem\#.V8zRu JgrK01. Acesso em: 03 de Setembro de 2016.

CALIXTO M. F. et al. Prevalência de sintomas osteomusculares e suas relações com o desempenho ocupacional entre professores do ensino médio público. Cadernos de Terapia Ocupacional, v. 23, n. 3, p. 533-542, 2015.

CEZAR-VAZ, M. R. et al. Mental health of elementary school teachers in southern Brazil: working conditions and health consequences. The Scientific World Journal, 2015.

CORTEZ.P. A. et al. A saúde docente no trabalho: apontamentos a partir da literatura recente. Cadernos de Saúde Coletiva, v. 25, n. 1, p. 113-122, 2017.

COSTA A. L.; FLAUSINO T. C. Prevalência dos distúrbios osteomusculares relacionados ao trabalho (DORTs) em maqueiros de um centro de reabilitação na cidade de Goiânia-GO. Revista Eletrônica Saúde e Ciência. v. 5, n. 1, 2015.

DAVOGLIO, T. R.; LETTNIN, C.; BALDISSERA, C. G. Avaliação da qualidade de vida em docentes brasileiros uma revisão sistemática. Pro-Posições, v. 3, n. 78, p. 145166, 2015.

ERICK, P. N.; SMITH, D. R. The prevalence and risk factors for musculoskeletal disorders among school teachers in Botswana. Occupational Medicine \& Health Affairs, v. 2, n. 4, 2013.

FERREIRA, R. C. et al. Transtorno mental e estressores no trabalho entre professores universitários da área de saúde. Trabalho, Educação e Saúde, v. 13, n. 1, p. 135155, 2015.

FLECK, Marcelo P.A. et al. Aplicação da versão em português do instrumento abreviado de avaliação da qualidade de vida "WHOQOL-bref". Revista de Saúde Pública, v. 34, n. 2, p. 178-83, 2000 
Programa de Pós-graduação em Educação, Universidade Federal do Ceará

Fortaleza-CE-Brasil

GERHARDT, G. 1.; SILVEIRA, T. D. (Orgs). Métodos de pesquisa. Planejamento e Gestão para o Desenvolvimento Rural da SEAD/UFRGS. Porto Alegre: UFRGS, 2009.

HOCHMAN, B. et al. Desenhos de pesquisa. Acta Cirúrgica Brasileira, v. 20, supl. 2, 2005.

IBGE. estimativa_ibge_2017.xIs. Disponível em: agenciadenoticias.ibge.gov.br. Acesso em 30 de agosto de 2017, 2017a.

IBGE. Pesquisa nacional de saúde, 2013: percepção do estado de saúde, estilos de vida e doenças crônicas: Brasil e grandes regiões / IBGE, Coordenação de Trabalho e Rendimento. - Rio de Janeiro: IBGE, 2014.

IBGE. Práticas de esporte e atividade física: 2015 / IBGE, Coordenação de Trabalho e Rendimento. - Rio de Janeiro: IBGE, 2017b.

INEP. Censo escolar da educação básica 2016. Brasília-DF, 2017.

IPEA. Professores da educação básica no brasil: condições de vida, inserção no mercado de trabalho e remuneração. Brasília: Rio de Janeiro: Ipea, 2017.

KARAKAYA, I. Ç. et al. Musculoskeletal problems and quality of life of elementary school teachers. International Journal of Occupational Safety and Ergonomics, v. 21, n. 3, 2015.

MARINHO, E. F. Estudo das principais queixas álgicas indicativas de DORT em professores de educação especial. Pós-graduação em Ortopedia e Traumatologia com ênfase em Terapia Manual - Faculdade Ávila, 2012.

MARQUES, F. Fatores associados à qualidade de vida de docentes da área da saúde. Revista Brasileira de Educação Médica, v. 40, n. 3, p. 452-460, 2016.

MANGO, M. S. M. et al. Análise dos sintomas osteomusculares de professores do ensino fundamental em Matinhos (PR). Fisioterapia em Movimento, v. 25, n. 4, p. 785-94, 2012.

MELO, B. F. et al. Estimativas de lesões por esforço repetitivo/distúrbios osteomusculares relacionados ao trabalho e indicadores de vigilância em saúde do trabalhador: um desafio para os serviços de saúde. Revista Baiana de Saúde Pública. v. 39, n. 3, p. 570-583, 2015.

MESARIA S.; JAISWAL N. Musculoskeletal disorders among teachers residing in various nations: a review. Research Journal of Recent Sciences, v. 4, p. 23-27, 2015.

MOREIRA, A. S. G. Qualidade de vida dos professores do ensino fundamental de escola da rede pública. (TCC). UEPB. Campina Grande, PB, 2015.

MOREIRA, A. S. G.; SANTINO, T. A.; TOMAZ, A. F. Qualidade de vida de professores do ensino fundamental de uma escola da rede pública. Ciencia e Trabajo, v. 55, n. 
ORGANIZAÇÃO MUNDIAL DA SAÚDE (OMS). Quality of life assessment: position paper from the World Health Organization. Social Science Medicine, v. 41, n. 10, p. 1403-1409, 1945.

PEREIRA, E. F.; TEIXEIRA, C. S.; LOPES, A. S. Qualidade de vida de professores de educação básica do município de Florianópolis, SC, Brasil. Ciência \& Saúde Coletiva, v. 18, n. 7, p. 1963-1970, 2013.

PINHEIRO, F. A. et al. Validação do Questionário Nórdico de Sintomas Osteomusculares como medida de morbidade. Revista de Saúde Pública, v. 36, n. 3, p. 307-12, 2002.

PIMENTEL, B N. et al. Percepções do ruído, saúde auditiva e qualidade de vida de professores de escolas públicas. Audiololgy - Communication Research, v. 21, e1740, p. 1-7, 2016.

ROCHA, R. E. R. et al. Sintomas osteomusculares e estresse em professores. Fisioterapia e Pesquisa, v. 24, n. 3, p. 259-266, 2017.

VASCONCELOS, L. B. et al. Qualidade de vida relacionada à saúde: análise dimensional do conceito. Investigação Qualitativa em Saúde: Avanços e Desafios, v. 3, p. 226-238, 2020.

WHOQOL Group. The World Health Organization quality of life assessment (WHOQOL): development and general psychometric properties. Social Science and Medicine, v. 46, p. 1569-85, 1988.

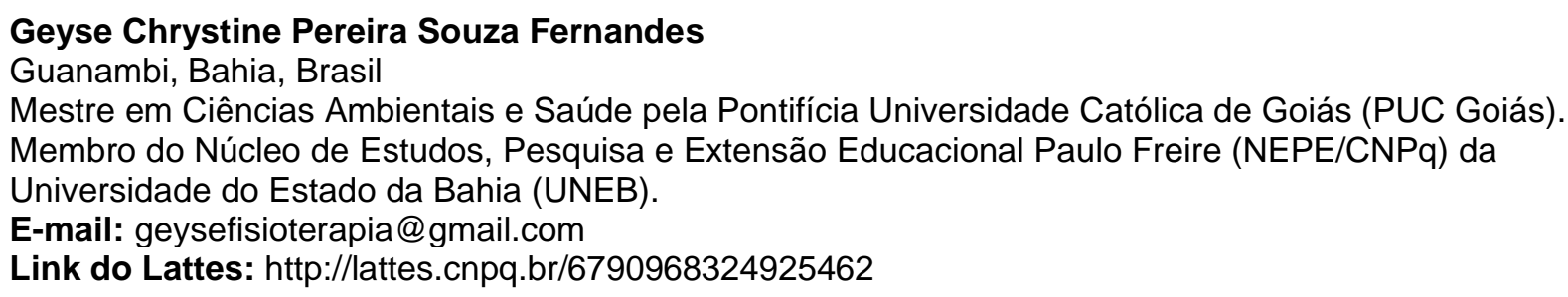

\section{Rogério José de Almeida}

Goiânia, Goiás, Brasil

Possui Graduação em Ciências Sociais pela Universidade Federal de Goiás, Mestrado em Sociologia pela Universidade Federal de Goiás, Doutorado em Sociologia pela Universidade de Brasília e, atualmente, Pós-Doutorando em Ciências da Saúde pela Universidade Federal de Goiás. Professor Adjunto do Curso de Medicina da PUC Goiás e do Programa de Pós-Graduação em Ciências Ambientais e Saúde PPGCAS da PUC Goiás. Coordenador do Comitê de Ética em Pesquisa - CEP/PUC Goiás. Professor Adjunto e Coordenador de Pesquisa e Extensão da Faculdade da Política Militar - FPM. Autor do livro Gastroplastia e a Reconstrução da Identidade e organizador do livro Goiás e a (Pós) Modernidade: Dimensões e Reflexões. Tem experiência na área de Sociologia, Antropologia e Metodologias Ativas, com ênfase em Sociologia da Cultura, Sociologia da Medicina, Sociologia da Saúde e do Corpo e Saúde Coletiva, atuando principalmente na linha de pesquisa Sociedade, Ambiente e Saúde e com temas que fazem interseções entre as Ciências Sociais e os processos saúde-doença.

E-mail: rogeriopucgo@gmail.com

Link do Lattes: http://lattes.cnpq.br/5504604536035282 
Recebimento: 05/07/2020

Aprovação: 29/12/2020

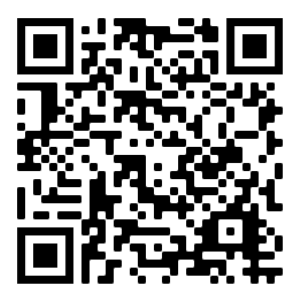

Q. Code

\section{Editores-Responsáveis}

Dr. Enéas de Araújo Arrais Neto, Universidade Federal do Ceará, UFC, Ceará, Brasil

Dr. Sebastien Pesce, Universidade de Orléans, França 